\title{
Síndrome 3-M: descripción clínica-radiológica. Caso clínico
}

\author{
MARIANA ARACENA A. ${ }^{1-2}$, LAURA MACHO F. ${ }^{3}$, VALERIE CORNIER-DAIRE ${ }^{4}$, \\ CELINE HUBER-LEQUESNE ${ }^{4}$, GEN NISHIMURA Y. ${ }^{5}$ \\ 1. Pediatra, Unidad de Genética, Hospital Dr. Luis Calvo Mackenna. \\ 2. Pediatra, Sección de Neurología- Genética y Enfermedades Metabólicas, División de Pediatría, Pontificia Universidad \\ Católica de Chile. \\ 3. Radióloga Pediátrica, Unidad Radiología Hospital Dr. Luis Calvo Mackenna. \\ 4. Department of Genetics, Université Paris Descartes, INSERM U781, Hospital Necker Enfants-Malades, Paris, France. \\ 5. Radiologist-in-Chief, Department of Pediatric Imaging, Tokyo Metropolitan Children's Medical Center, Japan.
}

\begin{abstract}
3-M syndrome: clinical and radiological description. Case report

Introduction: $3 \mathrm{M}$ syndrome combines severe prenatal and postnatal growth delay, facial dysmorphism (resembles melancholy facies) and radiological abnormalities. It is a rare disease with 200 cases reported so far. " $3 \mathrm{M}$ " refers to the initials of the three authors who first described this syndrome. The characteristic facial features are: relatively large head, dolichocephaly, frontal bossing, a triangular face, pointed chin, upturned nose, full lips, full eyebrows, long philtrum and hypoplastic midface. radiological findings which appear with age, include slender long bones and ribs and tall vertebral bodies. $3 \mathrm{M}$ syndrome is transmitted as an autosomal recessive trait and is genetically heterogeneous. Objective: Report the clinical case of a girl, now 10 years of age, diagnosed with the syndrome. Case report: An infant girl, 15 months old, was referred to Genetics Clinic due to severe short stature, dysmorphic features and malformations. Her clinical and radiological follow-up led to propose and confirm this diagnosis. Conclusion: Sometimes only longitudinal monitoring of patients with severe short stature evidence abnormalities suggesting a specific diagnosis. The right diagnosis results in suitable clinical care and genetic counseling to parents.
\end{abstract}

(Key words: McKusick-Malvaux-Miller Syndrome (3M), Fetal growth retardation, Dwarfism, Spine abnormalities).

Rev Chil Pediatr 2012; 83 (6): 587-594

\section{RESUMEN}

Introducción: El síndrome 3M combina retardo de crecimiento prenatal y postnatal severo, dismorfias faciales (semeja facies "melancólica") y anomalías radiológicas. Es una enfermedad infrecuente de la que hasta el

Recibido el 13 de marzo de 2012, devuelto para corregir el 16 de abril de 2012, segunda versión el 28 de junio de 2012 , aceptado para publicación el 06 de agosto de 2012.

Consentimiento informado de fotografías clínicas: presentado conforme.

Este trabajo cumple con los requisitos sobre consentimiento /asentimiento informado, comité de ética, financiamiento, estudios animales y sobre la ausencia de conflictos de intereses según corresponda.

Correspondencia a:

Dra. Mariana Aracena Alvarez

E-mail:maracen@gmail.com 
momento se han descrito alrededor de 200 casos. "3M" se refiere a las iniciales de los tres autores que describieron este síndrome. Los rasgos faciales característicos son: cabeza relativamente grande, dolicocefalia, abombamiento frontal, cara triangular, mentón prominente, nariz antevertida, labios gruesos, cejas gruesas, surco nasolabial largo e hipoplasia de tercio medio de cara. Los hallazgos radiológicos, que van apareciendo con la edad son costillas y huesos largos finos y delgados, y cuerpos vertebrales altos. El síndrome $3 \mathrm{M}$ se transmite como un rasgo autosómico recesivo y es genéticamente heterogéneo. Objetivo: Descripción del caso clínico de una niña actualmente de 10 años de edad en el que se confirmó este síndrome. Caso clínico: Niña referida a Genética a los 15 meses de vida, por talla baja severa, dismorfias y malformaciones. Su seguimiento clínico y radiológico permitió plantear y confirmar este diagnóstico. Conclusión: En ocasiones sólo el seguimiento longitudinal de pacientes con talla baja severa permite que se evidencien alteraciones sugerentes de un diagnóstico específico. La certificación del diagnóstico favorece un adecuado manejo clínico y consejería genética a los padres.

(Palabras clave: Miller-McKusick-Malvaux-Syndrome (Síndrome 3M), retardo de crecimiento fetal, enanismo, alteraciones de columna vertebral).

Rev Chil Pediatr 2012; 83 (6): 587-594

\section{Introducción}

En ocasiones los pediatras se ven enfrentados a pacientes con retardo de crecimiento intrauterino severo y talla baja subsecuente. Las posibilidades diagnósticas son variadas y entre ellas se encuentran los síndromes malformativos, los que individualmente son muy infrecuentes, por lo que no se sospecha su diagnóstico. Una de estas condiciones es el Síndrome 3-M (OMIM 273750), denominado así por las iniciales de los tres primeros autores que lo delinearon en $1975^{1}$. Se han reportado alrededor de 200 casos $^{2}$.

Se caracteriza por rasgos faciales distintivos, retardo severo del crecimiento pre y postnatal, inteligencia y función endocrina normal ${ }^{1,3}$. Presentan además alteraciones esqueléticas destacando huesos tubulares largos, finos, con cuerpos vertebrales altos por disminución de la distancia anteroposterior y transversal de éstos. Otras de las manifestaciones esqueléticas son hiperlaxitud articular, luxaciones articulares, escápulas aladas y pies planos ${ }^{4}$.

El síndrome 3-M es una condición autosómica recesiva y se debe a mutaciones en al menos tres genes: $C U L 7^{5}, O B S L 1^{6,7}, C C D C 8^{8}$, lo que evidencia su heterogeneidad genética.

Reportamos hallazgos clínicos, radiológicos y evolución del primer caso chileno confirmado, en el que se encontró una mutación en estado homocigoto en el gen OBSL1. Se presenta una revisión exhaustiva de la literatura.

\section{Caso clínico}

Paciente de sexo femenino, actualmente 10 años de edad, nacida el 29 de noviembre de 2001. Hija única de padres jóvenes, sanos, consanguíneos, de talla normal. Embarazo controlado, no se reportan alteraciones ecográficas prenatales. Cesárea a las 36 semanas de gestación. RNPT 36 sem PEG, peso RN: $1,7 \mathrm{~kg}$; talla RN: $36,5 \mathrm{~cm}$ y cc: $24,5 \mathrm{~cm}$. Al nacer destaca por su talla corta, cabeza relativamente grande, ojos prominentes, hipotonía e hiperlaxitud articular. Se plantea displasia esquelética, no se evidenció alteraciones en estudio radiológico completo de recién nacido. Hormonas tiroideas y Ecografía encefálica normal. En período lactante presenta marcado retraso pondoestatural y disnea al alimentarse. Ecocardiografía revela defectos septales auriculares múltiples, los que se reparan quirúgicamente a los 18 meses de vida. Ecografía abdominal muestra riñones fusionados.

Evaluada en Clínica Genética a los 17 meses de vida. Se evidencia talla baja desproporcionada, macrocefalia relativa, frontal alto, no abombado, tronco corto, abdomen bultuoso. Peso: $6,4 \mathrm{~kg}(-5,57 \mathrm{DS})$; talla: $63,2 \mathrm{~cm}(-4.9$ DS); cc: $47,4 \mathrm{~cm}(0,82 \mathrm{DS})$. Facies oval, puente nasal bajo, ojos prominentes, con ligera disposición antimongoloide, escleras azuladas, extremidades superiores cortas, con manos pequeñas, y marcada hiperlaxitud articular (figuras 1,2 y 3 ). Piel suave aterciopelada e 


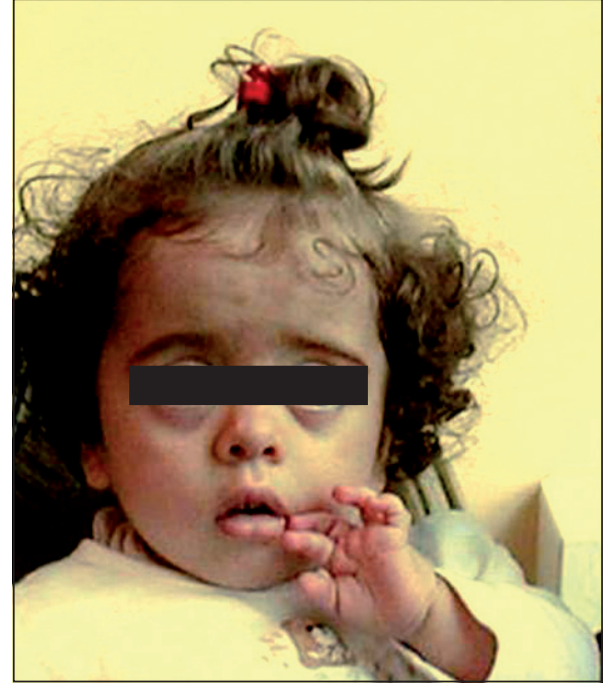

Figura 1. Macrocráneo, facies oval, frontal alto, ojos prominentes, narinas antevertidas.

hiperelástica. Evoluciona con talla baja severa, leve retraso del desarrollo de predominio motor, arqueamiento proximal de antebrazos, y aparición de escoliosis a los 24 meses. Esta progresa clínica y radiológicamente hacia cifoescoliosis dorsal, por lo que se indica corsé de Milwaukee, lo que estabiliza la escoliosis. En los estudios radiológicos completos realizados en edad preescolar y escolar no se observó alteración radiológica que sugiriese un diagnóstico. Estudio de hormonas tiroideas e IGF1 normal.

Tratándose de la primera hija de padres consanguíneos, que solicitaban consejería genética, y clínica sugerente de displasia ósea se continuó presentando en reuniones dismorfológicas y radiológicas a fin de dilucidar su diagnóstico. En la reunión de ISDS (International Skeletal Dysplasia Society) del año 2009, uno de los autores (GN) repara en el incremento de la altura de las vértebras, lo cual aunado al fenotipo le llevó a postular Síndrome 3-M (figura 4). La secuenciación del gen OBSL1 en la paciente, en el Departamento de Genética, Universidad Paris Descartes, reveló una inserción/deleción en estado homocigoto en c.661-662delGGinsC p.Gly221Argfs*37, lo que confirmó este diagnóstico. Ambos padres resultaron heterocigotos para ella.

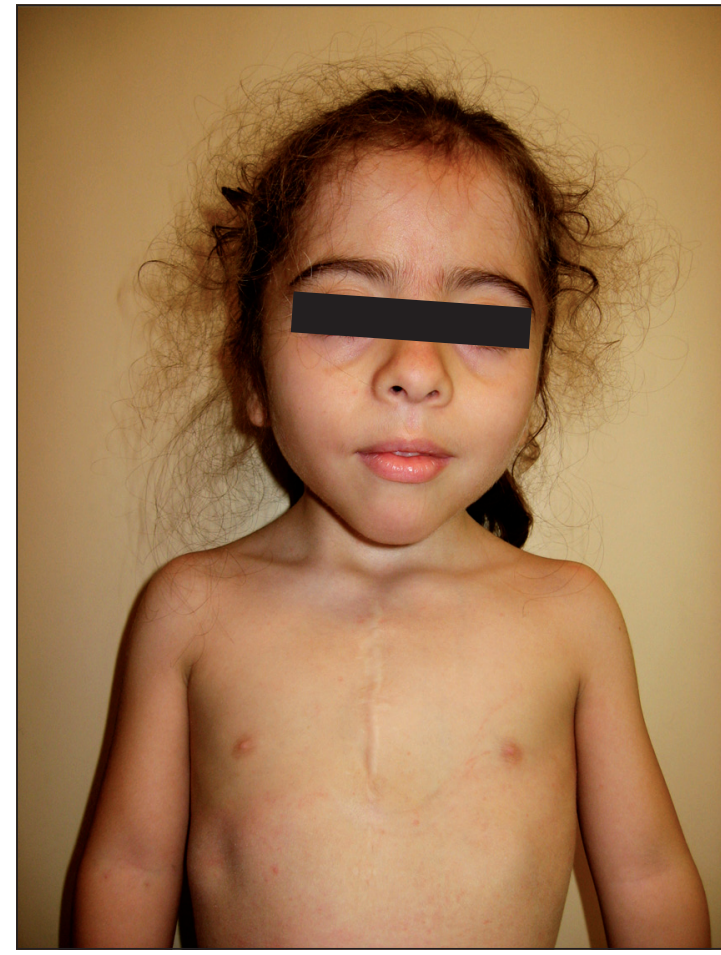

Figura 2. (Facies a los 6 años): Hipoplasia tercio medio, nariz con punta "carnosa", filtrum largo, labios gruesos, mentón puntiagudo.

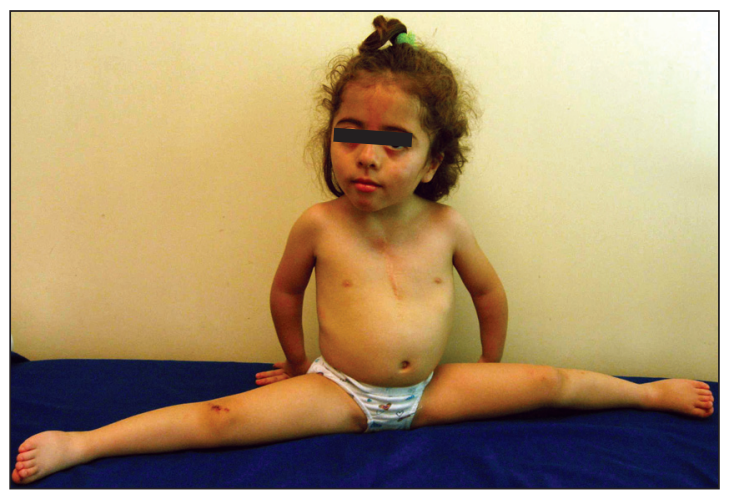

Figura 3. Hiperlaxitud articular, arqueamiento antebrazos.

Niña evoluciona con talla baja severa, en general es sana, asiste a escuela normal, y ha requerido apoyo psicológico para manejo de autoestima. En último control realizado a los 10 años 1 mes tenía una talla de $107 \mathrm{~cm}(-5,08$ DS), peso: $21,2 \mathrm{~kg}(-2,79 \mathrm{DS})$, con IMC de $18,5$ ( $+0,61 \mathrm{DS})$, con botón mamario y vello pubiano I. Su facies se ha tornado triangular, 


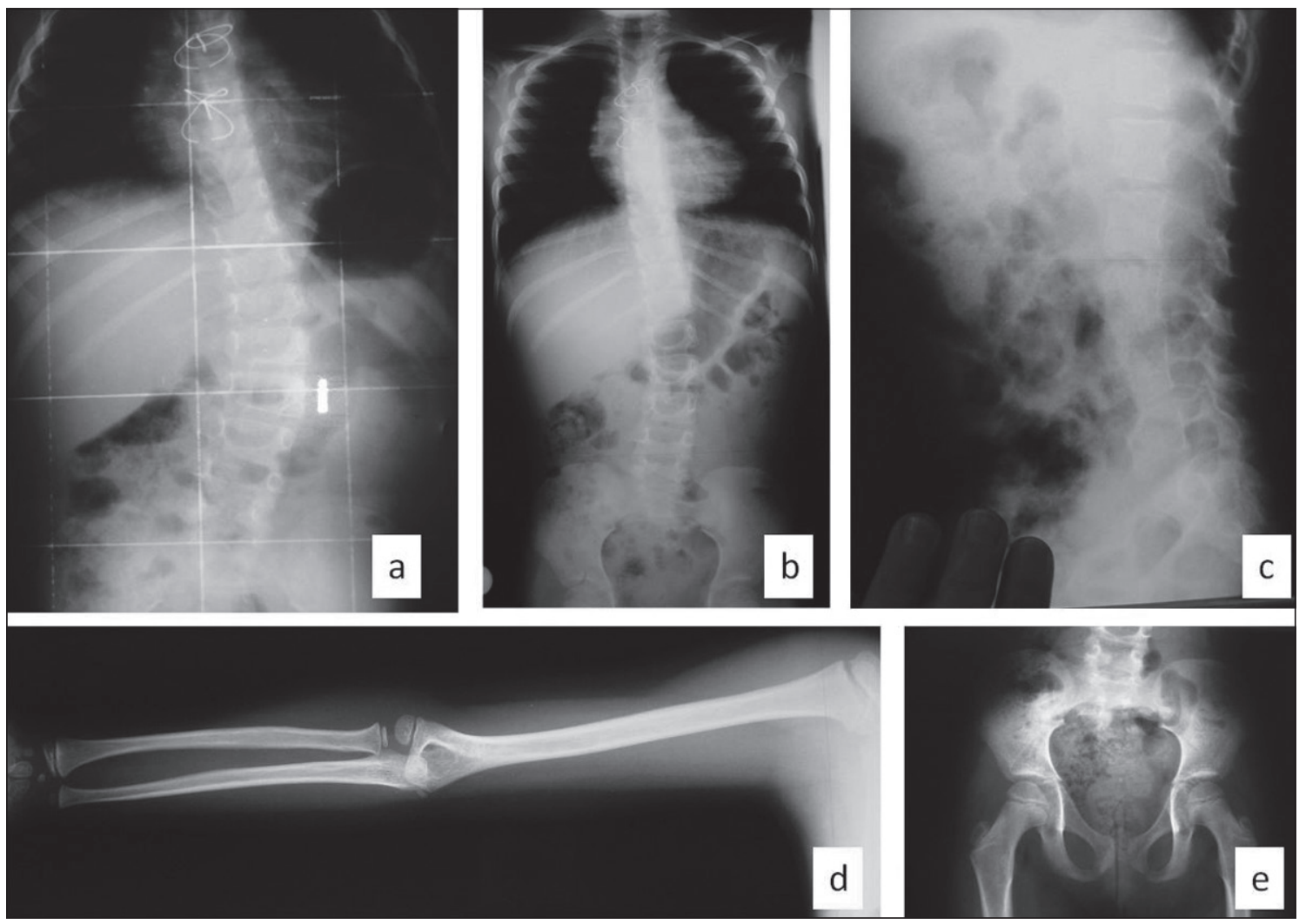

Figura 4. a) 2 a 8 m: escoliosis de convexidad izquierda; b-e corresponden a los 7 años 6 meses; las figuras b) costillas finas y horizontalizadas; c) cuerpos vertebrales altos con dismución del diámetro AP, particularmente en región lumbar; d) huesos largos delgados, diáfisis finas; e) pelvis pequeña, alas ilíacas pequeñas.

el cuello más trapezoide, con hombros cuadrados, antebrazos arqueados, e hiperlaxitud (7/9 según score Beihgton) $)^{9}$. En la figura 5 se muestra antropometría.

\section{Discusión}

En ocasiones resulta extremadamente difícil el diagnóstico específico en un paciente con retraso de crecimiento intrauterino severo y talla baja postnatal, toda vez que cada uno de los Síndromes Malformativos que pueden dar cuenta de ello, son infrecuentes, presentan superposición de rasgos clínicos y algunos signos específicos sólo aparecen con el tiempo. El Síndrome de Silver-Russell (SSR), el enanismo de Mulibrey, y el Síndrome de Dubowitz entre otros, son entidades con las cuales se debe hacer diagnóstico diferencial con el Sín- drome 3-M. El Síndrome de Silver Russell se caracteriza por Retardo de Crecimiento intrauterino (RCIU) acompañado de retraso post natal, pero a diferencia de los sujetos con Síndrome 3-M la talla baja es proporcionada, tienen facies triangular, abombamiento frontal, labios finos, asimetría corporal o de extremidades, clinodactilia, talones normales, y grados variable de dificultades de aprendizaje. No se reporta en ellos anomalías esqueléticas a excepción de las asimetrías ${ }^{10,11}$. El enanismo de Mulibrey también presenta retardo de crecimiento pre y postnatal, pero la talla al nacer no es tan corta como la observada en el Síndrome 3-M. Su facies es distinta ya que suele ser triangular, con frontal alto que semeja al cráneo con hidrocefalia, puente nasal bajo, lengua pequeña, manos relativamente grandes y presentan pigmentos amarillos en la retina. Desde el punto de vista radiológico pueden presentar silla tur- 


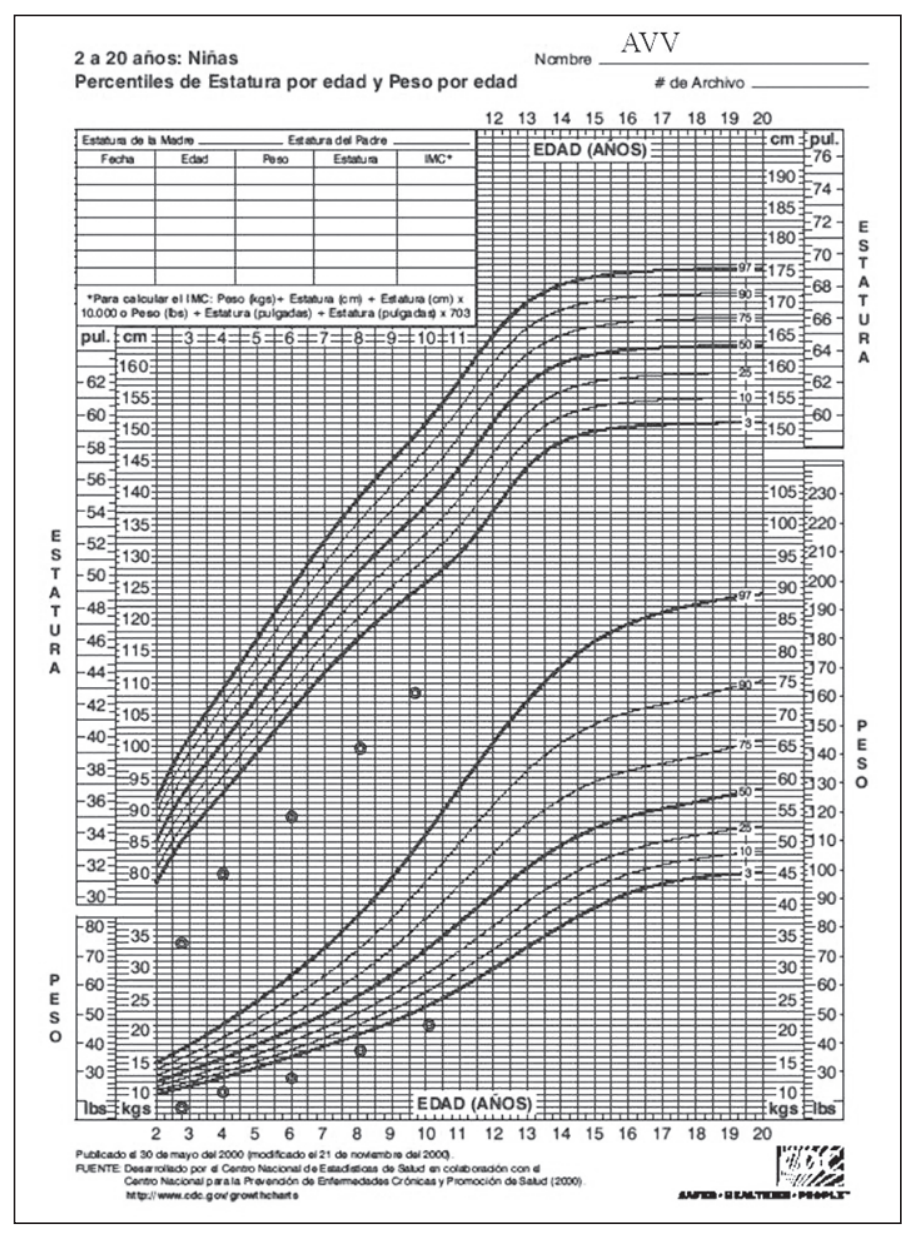

Figura 5. Curva antropométrica de paciente.

ca agrandada en forma de J y displasia ósea fibrosa. Sin embargo, lo más característico en el Enanismo de Mulibrey es el desarrollo de pericarditis constrictiva. Por último, el Síndrome de Dubowitz si bien tiene retraso pondoestatural pre y postnatal, se caracteriza por microcefalia, facies dismórfica con frontal huidizo, blefarofimosis y/o ptosis, eccema facial y discapacidad intelectual, rasgos que no presentan los pacientes con Síndrome 3-M. El fenotipo de Síndrome de Dubowitz es similar al de los pacientes con Síndrome Fetal alcohólico, sin embargo, en éstos últimos hay hipoplasia medio facial, filtrum liso, hirsutismo, discapacidad intelectual y problemas conductuales. En ellos está el antecedente de ingesta exagerada de alcohol durante el embarazo ${ }^{12}$.

La historia, evolución y fenotipo de nuestra paciente no se avenía a ninguno de estos síndromes y la convicción de estar frente a una displasia esquelética, muy probablemente autosómica recesiva por el antecedente de consan- guinidad de los padres instaba a sus médicos tratantes a continuar buscando un diagnóstico etiológico. La principal dificultad con la cual nos encontramos es que el signo radiológico que apuntó al diagnóstico de Síndrome 3-M, los cuerpos vertebrales altos, no se hizo evidente hasta la edad escolar y sólo fue reparado por radiólogo experto en displasias esqueléticas. Esta experiencia ratifica la utilidad de tener acceso a foros/redes de expertos en este caso de displasias esqueléticas, pero bien podría ser de síndromes malformativos en general, donde se exponen casos clínicos en los cuales no se tiene claridad en su diagnóstico.

Este Síndrome fue descrito por Miller y col en el año $1975^{1}$, siendo denominado 3-M por las iniciales de los tres primeros autores: Miller, McKusick y Malvaux. Un año más tarde Spranger y colli ${ }^{13}$, identificaron la misma condición. Desde sus primeras descripciones se señala herencia autosómica recesiva ${ }^{13-16}$. El rasgo más relevante en este síndrome es el severo retardo de crecimiento, de comienzo intrauterino, con cráneo de tamaño normal, el cual puede detectarse durante la segunda mitad del embarazo. En una serie publicada recientemente se señala que la talla promedio en recién nacidos de término fue de $41 \mathrm{~cm}(40-46 \mathrm{~cm})$, peso de $2200 \mathrm{~g}$ (2 000-3 $050 \mathrm{~g}$ ) y cc de $36 \mathrm{~cm}$ (32$37 \mathrm{~cm}$ ). No se observa crecimiento recuperacional postnatal, evolucionan con retraso de crecimiento severo y curvas de crecimiento tanto para hombres como mujeres muestran talla entre -4 y -6 DS, con talla final de $135,5 \mathrm{~cm}(128-143 \mathrm{~cm})^{3}$. A diferencia de otros síndromes con talla baja, tienen inteligencia normal y por lo general no presentan malformaciones mayores en otros 
sistemas, como la Comunicación Interauricular (CIA) encontrada en nuestra paciente, indicando que ésta es una condición que compromete principalmente el crecimiento ${ }^{2}$.

Durante los primeros años de vida se caracterizan por presentar una cabeza relativamente grande, cara redonda, frontal amplio, aplanamiento región malar, nariz corta con narinas antevertidas, punta de nariz carnosa, y labios gruesos ${ }^{16}$. Con el tiempo la facies se torna triangular con un mentón en punta y filtrum $\operatorname{largo}^{3}$, lo que se aprecia en la documentación fotográfica de nuestra paciente. El fenotipo facial varía considerablemente en los distintos pacientes descritos en la literatura ${ }^{17}$.

Rasgos adicionales son cuello corto, hombros cuadrados, tórax corto, deformación esternal, hiperlordosis lumbar ${ }^{17,18}$, hipermovilidad articular (generalizada o confinada a algunas articulaciones) $)^{1,15,19}$, escápulas aladas ${ }^{1,16}$, clinodactilia del quinto dedo ${ }^{13,18}$, pies planos, talones prominentes ${ }^{13,14,16}$ y luxación de cade$\operatorname{ras}^{4,14}$. Los talones prominentes, con protuberancia carnosa en la parte posterior de los talones, es una característica casi universal en los pacientes más pequeños con este síndrome ${ }^{10}$.

Una de las dificultades para formular este diagnóstico tempranamente es que el estudio radiológico completo del esqueleto es usualmente normal en el período de recién nacido, pudiendo observarse sólo un tórax ancho y costillas finas ${ }^{3}$. Con el tiempo, los huesos largos se tornan finos en la región diafisiaria y consecuentemente las metáfisis aparecen amplias ${ }^{16}$. Un hallazgo radiológico muy importante y que lo diferencia de otras displasias son los cuerpos vertebrales altos con disminución del diámetro antero-posterior y transverso, especialmente en la región lumbar ${ }^{16}$. Esto se va haciendo evidente con la edad pudiendo no observarse aún a los dos años de vida ${ }^{15}$. Esta situación también la vivenciamos en nuestra paciente.

El acortamiento anterior de los cuerpos vertebrales torácicos, la irregularidad superior e inferior de las plataformas, espina bífida oculta, huesos pélvicos pequeños, cubito corto, son otros rasgos de este síndrome. La cifoescoliosis torácica que desarrolló nuestra paciente, también es una complicación descrita en esta entidad. La edad ósea se encuentra levemente retrasada ${ }^{3}$.

La función gonadal en mujeres es normal, mientras que en los varones se ha documentado disfunción gonadal, con subfertilidad/infertilidad $^{17}$. Los niveles de Hormona de crecimiento (HC) son usualmente normales, y los de IGF-1 normales o bajos, mientras que la respuesta a terapia con Hormona de crecimiento es pobre. Estas observaciones sugieren que existe un grado de resistencia en el eje HC-IGF ${ }^{2}$. En un reporte se señala que el tratamiento con hormona de crecimiento en altas dosis pudiese ser efectivo en este síndrome ${ }^{17}$.

Se ha reportado siete casos de pacientes con síndrome 3-M y luxación congénita de las caderas $^{3,4}$, siendo un factor predisponente para ello la hiperlaxitud la cual está presente en más de la mitad de los casos descritos en la literatura. Este rasgo clínico fue notorio desde el período de neonato en nuestra paciente y en algún momento nos llevó a plantear Displasia Espondiloepimetafisiaria con Hiperlaxitud articular, hipótesis diagnóstica que luego se desechó al no encontrarse las alteraciones ni vertebrales ni epimetafisiarias características de esta entidad.

En la actualidad se conocen tres genes que se asocian a esta condición. El primero de ellos fue descrito el 2005 por Huber y col${ }^{5}$, quienes reportaron mutaciones patogénicas en el gen CUL7. Luego el año 2009 los mismos autores ${ }^{6}$ simultáneamente con otro grupo $^{7}$ encontraron mutaciones en el gen OBSL1. Recientemente se han reportado mutaciones en el gen $C C D C 8^{8,20}$ lo que evidencia su heterogeneidad genética. A la fecha, aproximadamente un $65 \%$ de las mutaciones descritas en este síndrome se encuentran en CUL7, mientras que un $30 \%$ se identifican en $O B S L 1^{10}$. No se ha encontrado correlación genotipo-fenotipo ${ }^{3}$.

El producto proteico CUL7 es central en la formación de un complejo enzimático ligasa E3 de ubiquitina, la cual integra la vía ubiquitina-proteosoma. Mediante esta vía numerosas proteínas son marcadas para su degradación, entre éstas, el sustrato 1 receptor insulina o IRS-1. No está del todo claro cómo las mutaciones en $C U L 7$ inciden finalmente en el fenotipo del síndrome 3-M, pero hay algu- 
nas observaciones importantes de señalar. Se conoce que CUL7 está involucrado en la degradación del gen supresor de tumor p-53, lo que sugiere que la deficiencia de CUL7 lleva al "upregulation" del gen p53, lo que se traduce en retardo de crecimiento y proliferación. También se ha observado en fibroblastos embrionarios de ratones "knockout" CUL7, una acumulación aberrante del sustrato 1 receptor insulina, lo cual podría desencadenar una regulación positiva de las proteínas transportadoras de IGF-1. Estas proteínas fijadoras/transportadoras de IGF-1 tienen gran afinidad con IGF-1, por lo que al unirse a éste en grandes cantidades, disminuye la actividad de IGF-1, con el consiguiente desmedro en el crecimiento ${ }^{20}$. OBSL1 es a su vez una proteína del citoesqueleto, la cual interactúa y estabiliza a CUL7, y el CCDC8 no tiene función conocida aún. Existe interacción física entre OBSL1 tanto con CUL7 y CCDC8, todo lo cual sugiere que estas tres proteínas son miembros de una misma vía regulatoria de crecimiento humano ${ }^{10}$.

En la literatura se ha reportado 27 familias OBSL1-deficientes, con 17 mutaciones distintas. Veinticinco familias tienen 2 alelos nulos, con 15 mutaciones diferentes (4 han sido mutaciones sin sentido, 6 con pequeñas deleciones, 4 con pequeñas inserciones y una con una mutación inserción/deleción), y en dos familias no se ha encontrado alelos nulos, siendo las dos mutaciones distintas (1 sin sentido y otra con una deleción de 3 bases) ${ }^{7,20-22}$. La alteración aquí reportada constituye una nueva mutación inserción/deleción en estado homocigoto, localizado en el exón 2: c.661-662delGGinsC (p.Gly221Argfs*37). La mutación de nuestra paciente determina un codón de término prematuro con la consiguiente proteína trunca.

\section{Conclusiones}

El diagnóstico de Síndrome 3-M se basa en el fenotipo, alteración severa del crecimiento y hallazgos radiológicos, siendo el diagnóstico definitivo la comprobación molecular ${ }^{12}$. Este examen se encuentra disponible a nivel asistencial en aún muy pocos laboratorios en el mundo. El conocimiento de los pediatras, endocrinólogos infantiles y genetistas clínicos de esta condición permitirá que frente a un paciente con estatura baja severa, lo consideren entre una de las posibilidades diagnósticas. El establecimiento de este diagnóstico evitará exámenes innecesarios, además de permitir una adecuada supervisión médica, y un consejo genético certero.

\section{Agradecimiento}

Se agradece a los padres de la paciente por su adhesión al control médico y por permitir la publicación de su caso clínico.

\section{Referencias}

1.- Miller JD, McKusick VA, Malvaux P, Temtamy S, Salinas $C$ : The 3-M syndrome: a heritable low birthweight dwarfism. Birth Defects Orig Artic Ser 1975; 11 (5): 39-47.

2.- Clayton PE, Hanson D, Magee L, et al: Exploring the Spectrum of 3-M Syndrome, a Primordial Short Stature Disorder of Disrupted Ubiquitination. Clin Endocrinol (Oxf) 2012 May 25.

3.- Huber C, Munnich A, Cormier-Daire V: The 3M syndrome. Best Pract Res Clin Endocrinol Metab 2011; 25 (1): 143-51.

4.- Badina A, Pejin Z, Odent T, et al: Hip dislocation in 3-M syndrome: risk of misdiagnosis. Clin Dysmorphol 2011; 20 (2): 114-6.

5.- Huber C, Dias-Santagata D, Glaser A, et al: Identification of mutations in CUL7 in 3-M syndrome. Nat Genet 2005; 37 (10): 1119-24.

6.- Huber C, Delezoide AL, Guimiot F, et al: A large-scale mutation search reveals genetic heterogeneity in $3 \mathrm{M}$ syndrome. Eur J Hum Genet 2009; 17 (3): 395-400.

7.- Hanson D, Murray PG, Sud A, et al: The primordial growth disorder 3-M syndrome connects ubiquitination to the cytoskeletal adaptor OBSL1. Am J Hum Genet 2009; 84 (6): 801-6.

8.- Hanson D, Murray PG, O'Sullivan J, et al: Exome sequencing identifies CCDC8 mutations in 3-M syndrome, suggesting that $\mathrm{CCDC} 8$ contributes in a pathway with CUL7 and OBSL1 to control human growth. Am J Hum Genet 2011; 89 (1): 148-53.

9.- Smits-Engelsman B, Klerks M, Kirby A: Beighton score: a valid measure for generalized hypermobility in chil- 
dren. J Pediatr 2011; 158 (1): 119-23, 123.e1-4.

10.- Hanson D, Murray PG, Black GC, Clayton PE: The genetics of 3-M syndrome: unravelling a potential new regulatory growth pathway. Horm Res Paediatr 2011; 76 (6): 369-78.

11.- Guven A, Cebeci AN: 3M syndrome: a report of four cases in two families. J Clin Res Pediatr Endocrinol 2011; 3 (3): 154-9.

12.- Holder-Espinasse M, Irving M, Cormier-Daire V: Clinical utility gene card for: $3 \mathrm{M}$ syndrome. Eur J Hum Genet 2011; 19 (9): 10.1038/ejhg.2011.32. Epub 2011 Mar 2.

13.- Spranger J, Opitz JM, Nourmand A: A new familial intrauterine growth retardation syndrome the "3-M syndrome”. Eur J Pediatr 1976; 123 (2): 115-24.

14.- Cantu JM, García-Cruz D, Sánchez-Corona J, Fragoso R, Hernández A, Nazara-Cazorla Z: 3-M slender-boned nanism. An intrauterine growth retardation syndrome. Am J Dis Child 1981; 135 (10): 9058.

15.- Winter RM, Baraitser M, Grant DB, Preece MA, Hall CM: The 3-M syndrome. J Med Genet 1984; 21 (2): 124-8.

16.- Hennekam RC, Bijlsma JB, Spranger J: Further delineation of the 3-M syndrome with review of the literature.
Am J Med Genet 1987; 28 (1): 195-209.

17.- van der Wal G, Otten BJ, Brunner HG, van der Burgt I: 3-M syndrome: description of six new patients with review of the literature. Clin Dysmorphol 2001; 10 (4): 241-52.

18.- Temtamy SA, Aglan MS, Ashour AM, Ramzy MI, Hosny LA, Mostafa MI: 3-M syndrome: a report of three Egyptian cases with review of the literature. Clin Dysmorphol 2006; 15 (2): 55-64.

19.- Van Goethem H, Malvaux P: The 3-M syndrome. A heritable low birthweight dwarfism. Helv Paediatr Acta 1987; 42 (2-3): 159-65.

20.- Al-Dosari MS, Al-Shammari M, Shaheen R, et al: 3M Syndrome: An Easily Recognizable yet Underdiagnosed Cause of Proportionate Short Stature. J Pediatr 2012 Feb 9.

21.- Huber C, Fradin M, Edouard T, et al: OBSL1 mutations in 3-M syndrome are associated with a modulation of IGFBP2 and IGFBP5 expression levels. Hum Mutat 2010; 31 (1): 20-6.

22.- Akawi NA, Ali BR, Hamamy H, Al-Hadidy A, Al-Gazali $L$ : Is autosomal recessive Silver-Russel syndrome a separate entity or is it part of the 3-M syndrome spectrum? Am J Med Genet A 2011; 155A (6): 1236-45. 